\title{
VALIDACIÓN Y MEJORA DE LAS SALIDAS NUMÉRICAS DE RADIACIÓN SOLAR PARA SU USO EN LA PRODUCCIÓN DE ENERGÍA SOLAR
}

\author{
Isabel Martínez Marco, José Luis Casado Rubio, María Aránzazu Revuelta \\ Menéndez, Cristina Robles González \\ Agencia Estatal de Meteorología (AEMET), C/ Leonardo Prieto 8, imartinezm@aemet.es
}

En los últimos años, ha aumentado el interés por mejorar la producción de las plantas operativas de energía solar a través de la mejora de la predicción meteorológica de la radiación solar. Esta predicción se realiza a partir de los valores suministrados por la modelización numérica del tiempo. En esta ponencia se expondrán varios trabajos realizados por AEMET, en colaboración con REE (Red Eléctrica Española), CENER (Centro Nacional de Energías Renovables) y DLR (German Aerospace Center) para la validación y mejora de la predicción de la energía solar utilizando las salidas de los modelos meteorológicos que emplea la Agencia.

En el marco del proyecto PreFlexMS (Predictable and Flexible Molten Salt plant, www.preflexms.eu), financiado por el programa europeo H2020, cuyo objetivo es incrementar la predecibilidad de las Plantas de Concentración Solar (CSP) que usan almacenaje de sales en fusión, AEMET ha montado un sistema de predicción a corto plazo (hasta dos días) junto a CENER y DLR. Recientemente, los modelos númericos de predicción del tiempo han comenzado a obtener, como salidas del modelo, la radiación solar directa (DNI) y varias publicaciones muestran que puede ser utilizada operacionalmente (Casado-Rubio et al, 2017; Fernández-Peruchena et al, 2017 ; Schroedter-Homscheidt et al, 2017; Troccoli et al, 2014). Para ello, se utiliza el modelo de alta resolución IFS (Integrated Forecast System) del ECMWF (European Centre for Medium-Range Weather Forecast) y el modelo de área limitada Harmonie-Arome (Hirlam Aladin Regional/Meso-scale Operational NWP In Europe)-(Applications of Research to Operations at Mesoscale) para la predicción determinista y el ensemble del ECMWF y el ensemble gSREPS (gamma Short Range Ensemble Prediction System) de AEMET para la predicción probabilística. El objetivo de nuestro trabajo es validar la fiabilidad y la exactitud de las salidas de DNI de estos modelos para alimentar el sistema de producción de las plantas solares. Se han calculado diferentes estadísticos usando diferentes periodos de acumulación, diferentes pasadas, etc. focalizándonos en las necesidades de los usuarios de las plantas solares. En esta ponencia, expondremos los primeros resultados y analizaremos la posibilidad de utilizarlos en un entorno operativo.

En el marco de la colaboración con REE, se ha desarrollado un postproceso para la predicción de DNI en las localizaciones de las centrales termosolares y fotovoltaicas en España. Se basa en la combinación de un modelo numérico de predicción que proporciona la información sobre la nubosidad junto a un modelo de transferencia radiativa y un modelo de predicción de contenido de aerosoles (Casado-Rubio et al, 2017). 
Los modelos meteorológicos operativos actuales predicen el contenido de vapor de agua y la nubosidad, aunque es una variable extremadamente difícil de predecir adecuadamente, pero no predicen el contenido de aerosoles en la atmósfera. Usan valores climatológicos constantes, por lo que no pueden predecir los cambios de radiación solar debidos a los aerosoles. Esto puede ser un grave problema especialmente para la DNI en España, dado que ésta se ve afectada muchos días por distintos tipos de aerosoles, y en particular por el polvo de origen sahariano.

Para incluir los cambios en el tiempo del contenido de aerosoles hemos desarrollado un algoritmo que utiliza el modelo de radiación libRadtran (www.libradtran. org) para predecir la radiación solar. LibRadtran es un modelo de radiación libre y gratuito que permite introducir como parámetro de entrada el contenido de aerosoles (obtenido a partir de modelos específicos de predicción de aerosoles), además de otros parámetros procedentes de modelos meteorológicos (como el vapor de agua), y calcula la radiación global, directa y difusa en puntos determinados.

Como modelo específico de predicción de aerosoles hemos utilizado el modelo resultante de los diferentes proyectos MACC (Monitoring Atmospheric Composition and Climate) (http://www.gmes-atmosphere.eu) que predice cinco tipos diferentes de aerosol (polvo atmosférico, sal marina, sulfatos, materia orgánica y black carbon) y el modelo regional de polvo atmosférico BSC-DREAM8b (Dust Regional Atmospheric Model) (http://sds-was.aemet.es) y como modelo meteorológico hemos empleado el modelo global IFS del ECMWF (http://www.ecmwf.int).

El método desarrollado es válido para todas las situaciones meteorológicas y proporciona una curva continua y estable de DNI. Este método ha sido validado frente a los valores observados de DNI en ocho estaciones localizadas en áreas con importantes recursos solares, tanto en situaciones de cielo despejado como con nubosidad, durante los años 2013 y 2014 donde hubo importantes intrusiones de polvo sahariano. Las salidas de DNI obtenidas muestran mejoras de un 5-12\% anuales frente a las mismas salidas obtenidas con el modelo global del ECMWF, que ha sido utilizado como referencia, en situaciones de alto contenido de aerosol (véase tabla 1). En todos los casos, los resultados indican que la nubosidad es el factor dominante en la atenuación de la radiación pero el contenido total de aerosoles no es despreciable e incluso, en determinadas situaciones con cielo despejado y elevados niveles de aerosol, se observan mejoras del 50\%. La combinación de las predicciones de ECMWF, LibRadtran y MACC proporciona los mejores resultados, aumentando la exactitud de la predicción de DNI en sucesos con alto contenido de aerosoles. El modelo regional BSC-DREAM8b muestra buenos resultados en eventos extremos de alto contenido de polvo, aunque las predicciones más realistas, válidas para cualquier tipo de aerosol, las proporciona el modelo MACC. 


$\begin{array}{llll}\text { Estación } & \text { Año } & \text { rRMSE (IFS) } & \text { rRMSE (IRt + MACC) }\end{array}$

$\begin{array}{llll}\text { Badajoz } & 2013 & 21,2 \% & 13,7 \% \\ \text { Madrid } & 2013 & 18,9 \% & 13,3 \% \\ \text { Murcia } & 2013 & 24,0 \% & 17,5 \% \\ \text { Badajoz } & 2014 & 19,5 \% & 14,5 \% \\ \text { Madrid } & 2014 & 19,0 \% & 15,0 \% \\ \text { Murcia } & 2014 & 23,6 \% & 17,4 \%\end{array}$

Tabla 1. Valores anuales del error cuadrático medio relativo rRMSE de las salidas de DNI obtenidas del modelo IFS del ECMWF o del método combinado LibRadtran+MACC en tres estaciones :

Badajoz, Madrid y Murcia durante los años 2013 y 2014 en situaciones de alto contenido de aerosol.

\section{Referencias}

- Casado-Rubio, J.L., Revuelta, M.A., Postigo M., Martínez-Marco, I. \& Yagüe, C., 2017: A Postprocessing Methodology for Direct Normal Irradiance Forecasting using Cloud Information and Aerosol Load Forecasts. Journal of Applied Meteorology and Climatology, 56, 1595-1608.

- Fernández-Peruchena, C., Gastón, M., Schroedter-Homscheidt, M., Kosmale, M., Martínez Marco, I., García-Moya, J. A., \& CasadoRubio J.L. (2017). Dynamic paths: Towards high frequency direct normal irradiance forecasts. Energy, 132, 315-323.

- Schroedter-Homscheidt, M., A. Benedetti, \& N. Killius, 2017: Verification of ECMWF and ECMWF/MACC's global and direct irradiance forecasts with respect to solar electricity production forecasts. Meteorologische Zeitschrift, 26, 1-19.

- Troccoli, A., \& J.-J. Morcrette, 2014: Skill of direct solar radiation predicted by the ECMWF global atmospheric model over Australia. Journal of Applied Meteorology and Climatolology, 53, 2571-2588. 